\title{
Perfiles de actividad física, obesidad, autoestima y relaciones sociales del alumnado de primaria: un estudio piloto con Self-Organizing Maps \\ Physical activity, obesity, self-esteem, and social relationship profiles of primary school students: a pilot study with Self-Organizing Maps \\ *Victor Cubas-Martinez, ${ }^{* *}$ Adrià Marco-Ahulló, ***Gonzalo Monfort-Torres, *Israel Villarrasa-Sapiña, *Alberto Pardo- Ibañez, *Xavier Garcia-Masso \\ *Universidad de Valencia (España) **Vall d’Hebron Research Institute (España) ***Florida Universitaria (España)
}

\begin{abstract}
Resumen. Las relaciones sociales son de vital importancia para el correcto desarrollo de los seres humanos, y junto con la actividad física, la obesidad y la autoestima forman un conjunto de factores que pueden ser capaces de retroalimentarse entre sí, pero que hasta donde los autores saben, solo se han analizado por separado. Por tanto, el principal objetivo de estudio será realizar un análisis multifactorial que incluya todas las variables anteriormente mencionadas. Un total de 60 niños de edades comprendidas entre los 7 y los 13 años formaron la muestra de este estudio. Los instrumentos utilizados para las mediciones de las diferentes variables de estudio fueron el PAQ-C, la escala de autoestima de Rosenberg y una adaptación del Bull-S Test. Una vez recogidos todos los datos se realizó un análisis con SelfOrganizing Maps mediante el software Matlab R2008a y la SOM toolbox para Matlab. Los resultados obtenidos en este trabajo muestran la repercusión del Índice de Masa Corporal sobre los niveles de autoestima y las relaciones sociales, y la posible retroalimentación mutua entre estos dos últimos factores.
\end{abstract}

Palabras clave: niños, perfiles, obesidad, relaciones sociales, autoestima y actividad física

\begin{abstract}
Social relationships are vital for the proper development of humans, and along with physical activity, obesity, and self-esteem, they form a set of factors that may be able to feed each other; however, as far as the authors know, they have only been analyzed separately. Therefore, the main objective of this study is to carry out a multifactorial analysis that includes all the aforementioned variables. A total of 60 children between 7 and 13 years of age formed the sample of this study. The instruments used for the measurements of the different study variables were the PAQ-C, the Rosenberg self-esteem scale, and an adaptation of the Bull-S Test. Once all the data was collected, an analysis with Self-Organizing Maps was made, using the Matlab R2008a software and the SOM toolbox for Matlab. The results obtained in this work show the repercussion of Body Mass Index on the levels of self-esteem and social relations, and the possible mutual feedback between the last two factors.
\end{abstract}

Key words: children, profiles, obesity, social relationships, self-esteem, physical activity.

\section{Introducción}

Existen una serie de factores que según la literatura científica son influyentes de cara al desarrollo social de las personas en edad escolar, y el presente trabajo se centra en analizar algunos de ellos: el exceso de peso, la realización de actividad física (AF), el sexo y la autoestima.

En cuanto al exceso de peso es necesario señalar que en 2012 se estableció una prevalencia de sobrepeso y obesidad en niños y jóvenes de entre 8 y 17 años del 38.6\% de la población española, llegando a un $45 \%$ en niños de entre 8 y 13 años (Sánchez-Cruz, Jiménez-Moleón, FernándezQuesada, \& Sánchez, 2013). Independientemente de las causas, parece claro que la obesidad conlleva ciertos factores psicológicos que desempeñan un papel relevante en las relaciones (Bersh, 2006; Cabello Garza \& Zúñiga Zárate, 2007), influyendo en la aparición de un déficit de habilidades sociales (Lacunza, Caballero, Salazar, Sal, \& Filgueira, 2013).

Según Strauss, Rodzilsky, Burack, \& Colin (2001), existe una correlación significativa entre la realización de AF, la cantidad de AF practicada y las relaciones sociales, siendo estas más positivas cuando la cantidad de AF es mayor. Asimismo, existe evidencia de que una AF frecuente mejora de manera importante la forma física y la salud de los jóvenes, siendo este un aspecto preventivo respecto al riesgo de padecer sobrepeso y/o obesidad (Larouche, Boyer, Tremblay,

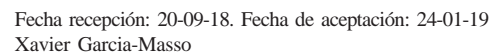

Fecha recepción: 20-09-18. Fecha de aceptación: 24-01-19 xavier.garcia@uv.es
\& Longmuir, 2014), en relación al factor comentado anteriormente.

El sexo también ha sido señalado como un factor que incide en la forma de relacionarse en el ámbito escolar (Coie, Dodge, \& Coppotelli, 1982; Peèjak, Puklek Levpušèek, Valenèiè Zuljan, Kalin, \& Peklaj, 2009); concretamente se ha observado que cada uno de los sexos presenta una conducta diferente a la hora de socializarse. Por ejemplo, los niños muestran un nivel más alto de agresión física que las niñas, en cambio, estas muestran un nivel más alto de agresión relacional y también un nivel más alto de competencia social (Cillessen \& Mayeux, 2004; Crick \& Grotpeter, 1995). Además, el número de niños agresores es más elevado que el de niñas, y sus agresiones tienen como objetivo a iguales del mismo sexo (Garaigordobil \& Antonio Oñederra, 2009).

La autoestima es el factor más influyente en las relaciones, ya que los niños que perciben un mayor apoyo de sus profesores y/o iguales manifiestan más interés y motivación por las actividades realizadas y las de los demás (Ochoa, 2001). Este aspecto despierta preocupación en algunos autores, los cuales hablan de los niveles de autoestima como una buena manera de identificar la vulnerabilidad social, siendo los sujetos con una baja autoestima los que corren un mayor riesgo de exclusión social (Wærdahl, Nilsen, Svarstad, \& Jentoft, 2017).

El presente trabajo tiene como principal objetivo establecer las relaciones multifactoriales existentes entre los conceptos de relaciones sociales, composición corporal (i.e., obesidad o sobrepeso), AF y autoestima en el alumnado de Educación Primaria. Hasta donde sabemos, estas variables 
influyen sobre las relaciones sociales, pero no conocemos la influencia de cada una de ellas cuando son tratadas conjuntamente. Por ello, con este estudio se ha intentado establecer perfiles de niños y niñas comprendidos entre 7 y 13 años, de modo que se puedan conocer las características más importantes para tener una mejor o peor interacción social.

\section{Material y métodos}

\section{Participantes}

La muestra para la realización de este estudio estuvo compuesta por niños y niñas escolarizados comprendidos entre los 7 y 13 años. De un total de 87 alumnos potenciales, 60 fueron finalmente los que participaron. Los criterios de exclusión fueron: i) negación a la participación en el estudio por parte de sus representantes legales; ii) no entregar el documento de consentimiento informado firmado.

Tabla 1.

\begin{tabular}{|c|c|c|c|}
\hline Variable & Total $(\mathrm{n}=60)$ & Chicos $(n=27)$ & Chicas $(\mathrm{n}=33$ ) \\
\hline Edad (años) & $9,47(1,70)$ & $9,22(1,74)$ & $9,67(1,67)$ \\
\hline Peso $(k g)$ & $38,72(12,27)$ & $36,87(11,41)$ & $40,24(12,91)$ \\
\hline Altura $(m)$ & $1,43(0,11)$ & $1,42(0,11)$ & $1,44(0,1)$ \\
\hline IMC $(\mathrm{kg} / \mathrm{m} 2)$ & $18,52(3,73)$ & $17,84(3,43)$ & $19,07(3,93)$ \\
\hline Percentil & $61,35(29,75)$ & $57,81(29,06)$ & $64,24(30,44)$ \\
\hline
\end{tabular}

Previamente al inicio de la recogida de datos se obtuvo la aprobación del proyecto por parte del Comité Ético de la Universidad de Valencia. Todos los participantes entregaron el consentimiento informado correctamente cumplimentado y firmado por sus tutores legales antes de realizar los protocolos de medición. Todos los protocolos aplicados en esta investigación cumplieron con los requisitos explicitados en la Declaración de Helsinki de 1975 y sus posteriores revisiones.

\section{Procedimiento general}

El primer paso fue pedir autorización a dos centros escolares públicos para la realización de la investigación. Después de obtener el permiso y una vez que los tutores legales firmaron el consentimiento informado y voluntario para participar en el estudio, se administraron los cuestionarios y se procedió con la valoración de la composición corporal, la cual se realizó mediante una báscula de bioimpedancia (Tanita BC-601; TANITA Corporation, Maeno-Cho, Itabashi-ku, Tokyo, Japón) y un tallímetro (Seca 206; SECA, Alemania). De estas valoraciones se obtuvo el peso de los alumnos, su porcentaje de grasa, su altura y su índice de masa corporal (IMC).

Los instrumentos utilizados fueron el PAQ-C (Physical Activity Questionnaire for Children), la escala de autoestima de Rosenberg (Rosenberg Self-Esteem) y un test de elaboración propia diseñado para obtener sociogramas. En primer lugar, el PAQ-C fue el instrumento elegido para la medición de la AF de los participantes, ya que se trata de un test que ha sido utilizado en numerosos estudios y cuya validez y fiabilidad han estado demostradas tanto en su versión original como en su traducción al español (Kowalski, Crocker, \& Faulkner, 1997; Manchola-González, Bagur-Calafat, \& Girabent- Farrés, 2017). Este test, diseñado para el cálculo de esta variable en niños de 8 a 14 años está compuesto por 10 preguntas relacionadas con la práctica de AF durante los últimos 7 días, de las cuales, se utiliza el promedio de las 9 primeras para hallar la puntuación final. La escala utilizada para las respuestas a las preguntas del cuestionario es de tipo Likert de 1 a 5 , siendo 1 «nada de actividad física» y 5 «actividad física intensa». En segundo lugar, la escala de autoestima de Rosenberg (Rosenberg, 2015) se utilizó para hallar el grado de autoestima de los participantes. Esta escala es el instrumento más usado para medir la autoestima (Robins, Hendin, \& Trzesniewski, 2001), y consta de 10 items (5 positivos y 5 negativos). La escala utilizada para dar respuesta a los ítems del instrumento es de tipo Likert, y las posibles contestaciones de cada ítem van de 1 a 4 siendo 1 «poco de acuerdo» y 4 «totalmente de acuerdo». Una vez completado se obtiene una dimensión única sobre la autoestima como resultado de sumar la puntuación de los ítems (invirtiendo previamente los ítems negativos). Por último, se utilizó una adaptación del Bull-S: Test de evaluación de la agresividad entre escolares (Ramírez, 2014), en el que se realizaron preguntas donde los sujetos debían nombrar a sus compañeros, con el fin de obtener como resultado sociogramas. Este test consta de 12 preguntas donde las respuestas deben ser los nombres de hasta tres compañeros/as de la clase. Las preguntas eran casi en su totalidad de tipo social donde había unas de tipo positivo y otras de tipo negativo, (e.g. ¿con quién te gusta estar durante la práctica de AF?; ¿A quién no invitarías a tu fiesta de cumpleaños?). Las respuestas se clasificaban por orden de preferencia siendo el primero nombre el de mayor puntuación (3) y el tercero el de menor puntuación (1).

\section{Análisis de datos}

\section{Sociogramas}

Los datos obtenidos mediante los test diseñados para obtener sociogramas se emplearon para obtener los grafos de cada grupo. Para ello se aplicó la teoría de grafos, con la cual se pueden analizar los diferentes vínculos que existen entre los miembros de un grupo. En este caso, se utilizó el software Pajek (version 1.28; Batagelj and Mrvar, University of Ljubljana, Ljubljana, Slovenia) para obtener dos sociogramas de cada clase. Uno negativo que hacía referencia a las preguntas del test con carácter negativo (i.e., preguntas 2-4-6-8-10-12), y otro positivo que hacía referencia a las preguntas de carácter positivo (i.e., preguntas 1-3-5-7-911).

Una vez obtenidos los sociogramas se calcularon, para cada nodo (alumno/a), los siguientes parámetros que representan su importancia dentro de la red social:

- Input: número de veces que cada alumno era elegido por un compañero.

- Input ponderado: número de veces que cada alumno era elegido por un compañero multiplicado por la importancia en función del orden de elección (i.e., si era la primera elección el factor multiplicador era 3; 2 si era la segunda elección y 1 si era elegido como tercera opción).

- Centralidad Sabidussi: se calcula como la inversa de las distancias existentes entre un alumno y el resto de sus compañeros.

- Centralidad Freeman: es la suma de probabilidades de que la distancia más corta entre dos alumnos ( $\mathrm{a}$ y b) pase por el alumno en cuestión (c). 
Focos: son alumnos que saben elegir a aquellos alumnos que son más populares o impopulares (dependiendo de la orientación de la pregunta).

- Autoridades: son los alumnos que son elegidos por muchos focos.

\section{Self-Organizing Maps}

A posteriori se llevó a cabo un análisis con Self-Organizing Maps (SOM), con el cual se intenta transformar un patrón de señal de entrada de dimensiones arbitrarias en un mapa discreto de dos dimensiones, y realizar esta transformación adaptativa de una manera topológicamente ordenada. Este tipo de análisis se emplea habitualmente como herramienta para clasificar o encontrar relaciones entre una serie de variables que describen un problema determinado mediante redes neuronales competitivas no supervisadas.

Para ello, fueron utilizadas como variables de entrada todas las variables de los sociogramas ( 6 positivas y 6 negativas) junto con los datos extraídos referentes a la autoestima, la AF, las variables socio-demográficas y el IMC de los alumnos. El software empleado fue Matlab R2008a (Mathworks Inc., Natick, USA) y la SOM toolbox (versión 2.0 beta) para Matlab (Vesanto, Himberg, Alhoniemi, \& Parhankangas, 1999).

El proceso comienza con la construcción de una red de neuronas o nodos cuyo tamaño depende del número de casos que se incluyen en el análisis. La matriz de datos que hemos empleado como entrada tiene una longitud de 60 casos. En nuestro estudio la red o rejilla tuvo una forma rectangular con un tamaño de 7 x 6 neuronas de alto y ancho respectivamente. La forma de las neuronas fue hexagonal, así cada neurona tuvo un total de 6 neuronas vecinas.

Una vez determinado el tamaño y la forma de la rejilla, se asignó un valor de cada variable de entrada a cada una de los nodos o neuronas (i.e., inicialización). La inicialización del SOM se realizó de dos formas diferentes que explicamos a continuación. Por una parte, la inicialización aleatoria ocurre cuando los vectores de peso son inicializados con un valor aleatorio pequeño. Por otra parte, la inicialización lineal ocurre cuando los vectores de peso son inicializados de forma ordenada a lo largo de un subespacio lineal atravesado por los dos principales vectores propios (eigenvectors) de la serie de datos de entrada.

Los pesos asignados inicialmente se modifican a lo largo del proceso de entrenamiento. En este estudio se aplicaron dos algoritmos de entrenamiento diferentes (i.e. secuencial y batch). En la fase de entrenamiento cada una de las neuronas que componen la rejilla compite por ganar cada uno de los vectores de entrada o casos que componen la muestra. La neurona ganadora de cada caso es aquella cuya distancia Euclídea entre su vector de pesos y el vector de entrada es menor. Cabe destacar que los casos son normalizados entre 0 y 1 antes de comenzar el proceso de entrenamiento. Esto se hace así para que la escala de las variables no influya en el entrenamiento del SOM.

Este proceso competitivo da paso a un proceso adaptativo por el cual una vez asignado el vector de datos de entrada a una neurona, los pesos de la neurona ganadora y de las neuronas vecinas se modifican.

En la ecuación 1 se muestra el cálculo que se emplea durante el entrenamiento de la red neuronal. Como puede apreciarse los pesos después de cada iteración los pesos neuronales se ven modificados en función de las diferencias entre los pesos iniciales y el vector de entrada, la función de vecindad y la ratio de aprendizaje.

$w_{j}(n+1)=w_{j}(n)+\eta(n) h_{j, i(x)}(n)\left(x-w_{j}(n)\right)$ Ec. 1

Donde $w_{j}$ es el vector de pesos de la $\mathrm{j}^{\text {th }}$ neurona, $\eta$ es la ratio de aprendizaje, $h_{j, i(x)}$ es la función de vecindad y $x$ es el vector de entrada. La función de vecindad se emplea para que la neurona ganadora y sus vecinas más cercanas adapten sus pesos para asemejarse al vector de entrada en mayor medida que las neuronas más alejadas de la ganadora. Para el presente estudio se testaron cuatro funciones de vecindad diferentes: i. gaussian, ii. cut gaussian, iii. Epanechicov y iv. Bubble.

La ratio de aprendizaje es un valor alto durante las primeras iteraciones y va disminuyendo progresivamente hasta valores muy pequeños. De esta forma, al comienzo del entrenamiento se producen grandes cambios en los pesos de las neuronas que van modificándose de forma más discreta a medida que avanza el proceso.

Todo el proceso descrito se repite 100 veces. Esto se debe a que el resultado final del análisis depende de algunos procesos aleatorios (e.g. inicialización y orden de entrada de los vectores de entrada). Al repetir 100 veces todo el proceso se aumentan las probabilidades de encontrar la mejor solución al problema. Ya que empleamos dos métodos de entrenamiento diferentes, cuatro funciones de vecindad y dos métodos de inicialización se obtuvieron finalmente 1600 SOM (i.e. 100 x 2 × 4 × 2). Se seleccionó como el mejor aquel que mostró un menor producto entre el error de cuantización y el error topográfico. Por un lado, el error de cuantización expresa lo bien que representa el vector de pesos de las neuronas a aquellos casos que pertenecen a cada uno de las neuronas. Por otro lado, el error topográfico está relacionado con la posición y el valor de las neuronas. Este error será bajo cuando las neuronas próximas se parecen entre sí más que las que están más alejadas.

A pesar de que los SOM tienen por finalidad agrupar casos de características similares en la misma neurona (i.e. cada neurona representa en sí mismo un clúster), ocasionalmente, para establecer grupos más grandes de personas, se realiza un análisis clúster. En nuestro caso se empleó un método k-means, y se probaron entre 2 y 10 conglomerados. Se seleccionó el número de conglomerados que proporcionó un menor error de cuantización.

Por último, y con la intención de reforzar el análisis visual realizado, se hizo un análisis Kruskal-Wallis para comprobar el efecto del clúster sobre las variables de entrada. El seguimiento se realizó mediante pruebas U de Mann-Whitney usando el ajuste de Bonferroni. Se estableció un error de tipo I menor de $5 \%(p<0,05)$.

\section{Resultados}

En la figura 1 se puede observar el SOM de nuestro estudio con los 17 planos componentes equivalentes a las 17 variables estudiadas. Centrándonos en la figura, en la esquina superior izquierda se encuentra el mapa de golpeos. Este mapa de golpeos proporciona información acerca del núme- 
ro de sujetos que se ha asignado a cada neurona. Se observa que en la parte central hay un total de 13 neuronas que no tienen ningún sujeto asignado, mientras que las neuronas periféricas están casi todas pobladas. En este mapa de golpeo se pueden ver también los cinco clústeres que han surgido de nuestro estudio.

A modo de ejemplo pasamos a describir los dos clústeres que más han llamado la atención de los autores por ser aquellos con un comportamiento antagónico en cuanto a las variables de relación social. Utilizando estas descripciones como guía y analizando la figura 1 los lectores podrán extraer sus propias conclusiones sobre el resto de clústeres.

El clúster 4 muestra unos niveles bajos en cuanto a IMC, aunque saludables, al igual que un escaso nivel de AF. La autoestima de estos sujetos es alta y, además, en las preguntas positivas los sujetos de este clúster son los más nombrados por sus compañeros y, por el contrario, son los menos nombrados en las variables de preguntar negativas.

Por último, en el clúster 5 encontramos sujetos de edades bajas y mayoritariamente de sexo masculino. Estos sujetos presentan unos niveles altos de IMC a pesar de que son sujetos con altos niveles de AF. Si los comparamos con el resto de los clústeres, son los que más AF realizan. También son los más nombrados por sus compañeros en las variables

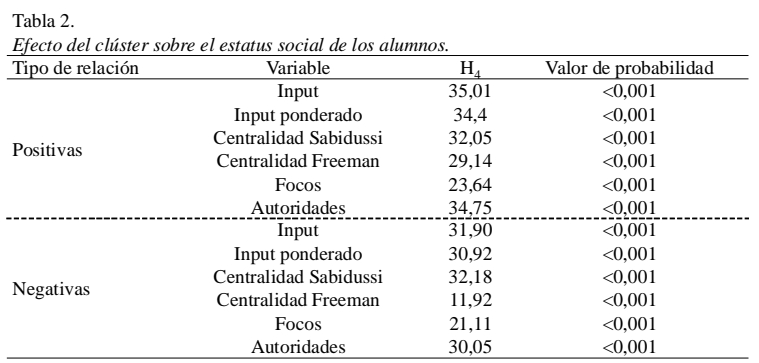

Tabla 3

Comparaciones entre los grupos de las diferentes variables.

\begin{tabular}{|c|c|c|c|c|}
\hline & Edad & Percentil & PAQ-C & Autoestima \\
\hline Clúster 1 & $\begin{array}{c}9,15 \\
(0,39)^{\mathrm{C} 2, \mathrm{C} 3, \mathrm{C} 4}\end{array}$ & $\begin{array}{c}48,32 \\
(11,09)^{\mathrm{C} 3, \mathrm{C} 5}\end{array}$ & $\begin{array}{c}3,08 \\
(0,07)^{\mathrm{C} 4, \mathrm{C} 3}\end{array}$ & $\begin{array}{c}33,72 \\
(0,83)^{\mathrm{C} 3, \mathrm{C5}}\end{array}$ \\
\hline Clúster 2 & $\begin{array}{c}9,77 \\
(0,23)^{\mathrm{C} 1}\end{array}$ & $\begin{array}{c}57,97 \\
(5,18)^{\mathrm{C} 5}\end{array}$ & $\begin{array}{c}3,00 \\
(0,06)\end{array}$ & $\begin{array}{c}33,62 \\
(0,52)^{\mathrm{C} 3, \mathrm{CS}}\end{array}$ \\
\hline Clúster 3 & $\begin{array}{c}9,85 \\
(0,08)^{\mathrm{C} 1}\end{array}$ & $\begin{array}{c}72,08 \\
(4,93)^{\mathrm{C} 4, \mathrm{C} 1}\end{array}$ & $\begin{array}{c}2,85 \\
(0,08)^{\mathrm{C} 1, \mathrm{C} 5}\end{array}$ & $\begin{array}{c}30,74 \\
(1,36)^{\mathrm{C} 1, \mathrm{C} 2, \mathrm{C} 4}\end{array}$ \\
\hline Clúster 4 & $\begin{array}{c}10,10 \\
(0,17)^{\mathrm{C} 1, \mathrm{CS}}\end{array}$ & $\begin{array}{c}46,02 \\
(9,19)^{\mathrm{C} 3, \mathrm{CS}}\end{array}$ & $\begin{array}{c}2,75 \\
(0,24)^{\mathrm{C} 1, \mathrm{C} 5}\end{array}$ & $\begin{array}{c}33,84 \\
(0,67)^{\mathrm{C} 3, \mathrm{C} 5}\end{array}$ \\
\hline Clúster 5 & $\begin{array}{c}9,43 \\
(0,33)^{\mathrm{C} 4}\end{array}$ & $\begin{array}{c}73,69 \\
(1,24)^{\mathrm{C} 4, \mathrm{C} 1, \mathrm{C} 2}\end{array}$ & $\begin{array}{c}3,15 \\
(0,07)^{\mathrm{C} 4, \mathrm{C} 3}\end{array}$ & $\begin{array}{c}32,19 \\
(0,64)^{\mathrm{C} 1, \mathrm{C} 2, \mathrm{C} 4}\end{array}$ \\
\hline
\end{tabular}

Los datos están expresados en media (desviación típica). ${ }^{\mathrm{C} 1}$ indica diferencias significativas con respecto al clúster $1 .{ }^{\mathrm{C} 2}$ indica diferencias significativas con respecto al clúster 2. ${ }^{\mathrm{C} 3}$ indica diferencias significativas con respecto al clúster 3 . $^{\mathrm{C} 4}$ indica diferencias significativas con respecto al clúster $4 .{ }^{\mathrm{C5}}$ indica diferencias significativas con respecto al clúster 5 .
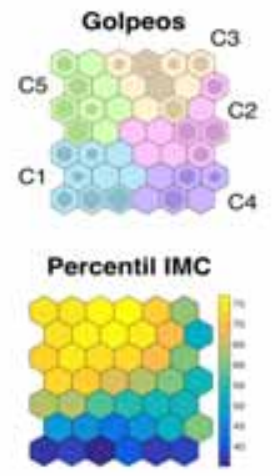

Input

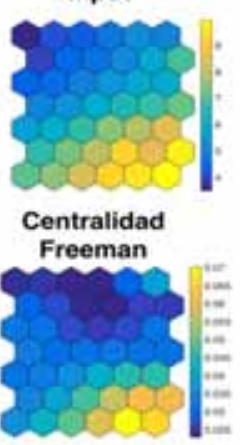

Input

Preguntas Positivas

\section{Preguntas} Negativas

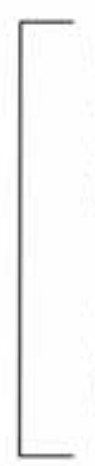

Edad

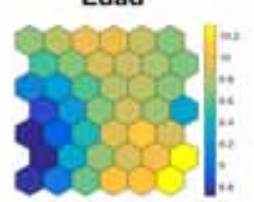

PAQ-C

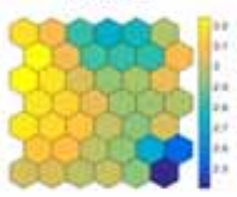

Input Ponderado

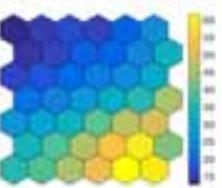

Focos

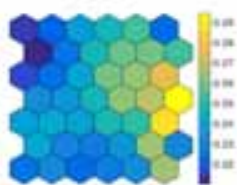

Input Ponderado

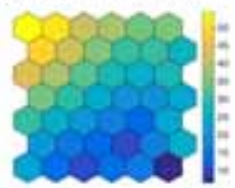

Focos

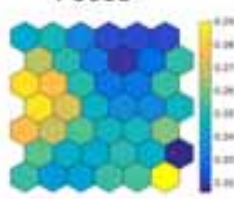

Sexo

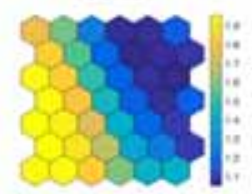

Autoestima

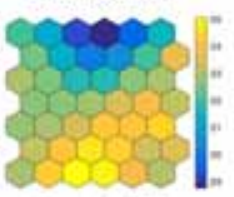

Centralidad

Sabidussi

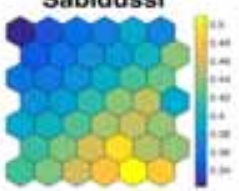

Autoridades

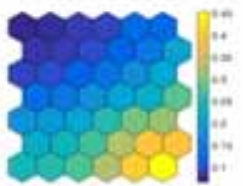

Centralidad

Sabidussi

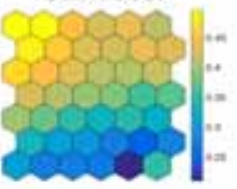

Autoridades

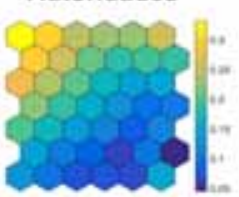

Figura 1. Mapa de golpeos y planos componentes del SOM. Chicas =1; Chicos =2.

de las preguntas negativas $\mathrm{y}$, por consiguiente, los menos nombrados en las positivas.

Se encontró un efecto del clúster sobre la edad de los sujetos $\left(\mathrm{H}_{4}=29,04 ; \mathrm{p}<0,001\right)$, sobre el percentil IMC de los sujetos $\left(\mathrm{H}_{4}=31,21 ; \mathrm{p}<0,001\right)$, sobre la práctica de $\mathrm{AF}\left(\mathrm{H}_{4}=30,18 ; \mathrm{p}<0,001\right)$, sobre la variable relacionada con autoestima $\left(\mathrm{H}_{4}=30,48 ; \mathrm{p}<0,001\right)$ y un efecto principal del clúster en todas las variables que informan sobre las relaciones sociales entre los alumnos (véanse los efectos de estas variables en la tabla 2 y las comparaciones por pares entre los clústeres en la tabla 3).

\section{Discusión}

La principal aportación de este trabajo de investigación ha sido el análisis multifactorial con SOM incluyendo las variables de AF, relaciones sociales, autoestima e IMC del alumnado durante la etapa de Educación Primaria. El presente estudio ha permitido determinar cinco perfiles diferentes de alumnado en función de estas variables. Con ello se ha observado que los perfiles de alumnos con un peso elevado muestran una menor autoestima y peores relaciones sociales. También se ha observado que tanto la autoestima como el IMC tienen una 
relación directa con las relaciones sociales. Por último, se ha concluido que la AF no se relaciona de forma directa con el peso, la autoestima y las relaciones sociales de los alumnos ya que los mapas de estas variables no muestran un patrón similar.

Recientes estudios como el realizado por DelgadoFloody, Carter-Thuillier, Jerez-Mayorga, Cofré-Lizama, \& Martínez-Salazar (2018) evidencian la existencia de una relación negativa entre los niveles de IMC y de autoestima, obteniendo la misma relación que la hallada en este estudio. Por otra parte, el sobrepeso y obesidad infantil se han convertido en la actualidad en un problema de salud pública tanto en Europa como en otros países desarrollados (Ahrens et al., 2014), muestra de ello son los preocupantes datos aportados por Lobstein et al. (2015) dónde exponen que, en unos 30 años en Estados Unidos el peso de cada niño ha aumentado $5 \mathrm{~kg}$ de media, llegando a establecerse los porcentajes de sobrepeso y obesidad en un 33\% de la población infantil. En cuanto a la marginación de los adolescentes con obesidad, nuestros resultados son similares a los que obtuvieron Strauss \& Pollack (2003) que afirmaron que los adolescentes que padecían sobrepeso solían estar socialmente aislados y en la periferia de sus redes sociales, y aunque aparentemente tenían el mismo número de amigos que los adolescentes con normopeso, sus nominaciones positivas eran menores que las de sus compañeros con normopeso, y sus nominaciones negativas más numerosas. Parece ser que el IMC es un factor influyente en la posición que se tiene en el mapa social del grupo en estas edades en el contexto escolar. Según el trabajo de Erickson, Robinson, Haydel, \& Killen (2000) el sobrepeso y la obesidad llevan aparejados además síntomas depresivos en chicas adolescentes, datos los cuáles parecen reforzar la información extraída de los clústeres de nuestro estudio, que señalan la misma relación entre chicas, IMC y nivel de autoestima. Dicho esto, los riesgos de sufrir estos altos niveles de IMC parece aumentar si los niños pertenecen a un nivel socioeconómico bajo (Gibbs \& Forste, 2014), y si a esto le sumamos que los niños y adolescentes que pertenecen a esta población presentan unos niveles más altos de comportamiento antisocial (Piotrowska, Stride, Croft, \& Rowe, 2015) hace que estos se establezcan como población con un alto riesgo de aislamiento social, por lo tanto, instituciones públicas y organismos científicos deberían de trabajar conjuntamente para establecer programas efectivos con los que conseguir que, indiferentemente del estatus socioeconómico del que provengan los niños puedan tener un correcto desarrollo físico y psicológico, sin que se les deje secuela alguna en su etapa adulta.

Si hablamos en cambio del factor AF, Tremblay, Inman, \& Willms (2000) relacionaron positivamente la cantidad de AF con una mayor autoestima; en nuestro estudio se han dado conclusiones similares, estableciéndose la misma relación en algunos grupos. En cambio, también han aparecido grupos con una práctica baja de AF y con un buen nivel de autoestima, lo cual nos indica que puede ser un factor a tener en cuenta para aumentar la autoestima, pero su déficit no señala necesariamente que esta sea baja. Kristjánsson, Sigfúsdóttir, \& Allegrante (2010), utilizando datos de los cuestionarios del estudio «Youth in Iceland» con una muestra final de 5810 adolescentes islandeses (51,7\% chicas, 48,3\% chicos) de entre 14 y 15 años, sí que hallaron que la autoestima se ve influida positivamente por la AF y negativamente por altos niveles de IMC. Además, hallaron una relación positiva entre los adolescentes con un IMC más bajo, unos buenos hábitos alimenticios y que practicaban más AF y un mayor rendimiento académico. Cabe señalar también que, aunque parece que la AF tiene relación con los niveles de autoestima, y por ello, una incidencia moderada sobre las relaciones sociales de los alumnos, según Cascales \& Prieto (2018) es importante ser cautos a la hora de enfocar las actividades físicas a practicar, controlando los ambientes competitivos donde los sujetos pueden verse más expuestos a conductas violentas. Por tanto, los profesionales de la AF que impartimos docencia debemos de elegir metodologías compatibles con estos objetivos, además de trabajar en optimizar el tiempo de compromiso motor en nuestras clases, ya que el tiempo dedicado a la Educación Física puede resultar escaso y/o insuficiente (2 horas semanales según el curriculum actual), y de esta manera poder aumentar el nivel de AF semanal realizado por los niños y niñas.(Heredia, Sánchez, Gallego, Zagalaz, \& Moral, 2018; Ortíz, Cid, Allepuz, \& Sánchez, 2018)

\section{Conclusiones}

Han sido muchos los estudios en niños y adolescentes que han intentado abordar los temas que se plantean en este estudio (i.e. AF, obesidad, autoestima y relaciones sociales) por separado, pero ninguno de los encontrados por los autores ha utilizado un análisis multifactorial teniendo SOM como herramienta de análisis. El hallazgo principal que se ha podido extraer de este trabajo y que se ha podido ver reforzado por los resultados de otros estudios, es la importancia del factor IMC para la autoestima y las relaciones sociales, y la posible retroalimentación mutua entre los dos últimos conceptos.

\section{Limitaciones}

Aunque este estudio presenta muchas fortalezas desde el punto de vista metodológico, cabe decir que la muestra del presente trabajo es relativamente escasa al tratarse de un estudio piloto, cosa que limita la generalización de los resultados obtenidos. Por tanto, con la vista puesta en futuras investigaciones, se debería continuar indagando en las cuestiones que abarca este estudio, con un análisis multifactorial de los datos y aumentando el tamaño de la muestra.

\section{Referencias}

Ahrens, W., Pigeot, I., Pohlabeln, H., De Henauw, S., Lissner, L., Molnár, D., ... IDEFICS consortium. (2014). Prevalence of overweight and obesity in European children below the age of 10. International Journal of Obesity (2005), 38 Suppl 2, S99107. https://doi.org/10.1038/ijo.2014.140

Bersh, S. (2006). La obesidad: aspectos psicológicos y conductuales. Revista Colombiana de Psiquiatría, XXXV(4), 537-546.

Cabello Garza, M. L., \& Zúñiga Zárate, J. G. (2007). Aspectos intrapersonales y familiares asociados a la obesidad: un análisis fenomenológico. Ciencia UANL, 10. Retrieved from http:// eprints.uanl.mx/1770/

Cascales, J. Á. M., \& Prieto, M. J. R. (2018). Incidencia de la 
práctica de actividad física y deportiva como reguladora de la violencia escolar (Incidence of the practice of physical and sporting activities as a regulator of school violence). Retos, O(35), 54-60.

Cillessen, A. H. N., \& Mayeux, L. (2004). Sociometric status and peer group behavior: Previous findings and current directions. In Children's peer relations: From development to intervention (pp. 3-20). Washington, DC, US: American Psychological Association. https://doi.org/10.1037/10653-001

Coie, J. D., Dodge, K. A., \& Coppotelli, H. (1982). Dimensions and types of social status: A cross-age perspective. Developmental Psychology, 18(4), 557-570. https://doi.org/ 10.1037/0012-1649.18.4.557

Crick, N. R., \& Grotpeter, J. K. (1995). Relational aggression, gender, and social-psychological adjustment. Child Development, 66(3), 710-722.

Delgado-Floody, P., Carter-Thuillier, B., Jerez-Mayorga, D., CofréLizama, A., \& Martínez-Salazar, C. (2018). Relación entre sobrepeso, obesidad y niveles de autoestima en escolares (Relation between overweight, obesity, and self-esteem levels in schoolchildren). Retos, 0(35), 67-70.

Erickson, S. J., Robinson, T. N., Haydel, K. F., \& Killen, J. D. (2000). Are overweight children unhappy?: Body mass index, depressive symptoms, and overweight concerns in elementary school children. Archives of Pediatrics \& Adolescent Medicine, 154(9), 931-935.

Garaigordobil, M., \& Antonio Oñederra, J. (2009). Un Análisis Del Acoso Escolar Desde Una Perspectiva De Género Y Grupo. An Analysis of Bullying at School from a Gender and Group Perspective., 15(2/3), 193-205.

Gibbs, B. G., \& Forste, R. (2014). Socioeconomic status, infant feeding practices and early childhood obesity. Pediatric Obesity, 9(2), 135-146. https://doi.org/10.1111/j.20476310.2013.00155.x

Heredia, C. M. R., Sánchez, A. J. L., Gallego, F. J. L., Zagalaz, J. C., \& Moral, P. V. (2018). Análisis del tiempo de clase en EF y propuestas para su optimización (Analysis of class time in physical education and proposals for optimization). Retos, O(35), 126-129.

Kowalski, K. C., Crocker, P. R. E., \& Faulkner, R. A. (1997). Validation of the Physical Activity Questionnaire for Older Children. Pediatric Exercise Science, 9(2), 174-186. https:// doi.org/10.1123/pes.9.2.174

Kristjánsson, A. L., Sigfúsdóttir, I. D., \& Allegrante, J. P. (2010). Health behavior and academic achievement among adolescents: the relative contribution of dietary habits, physical activity, body mass index, and self-esteem. Health Education \& Behavior: The Official Publication of the Society for Public Health Education, 37(1), 51-64. https://doi.org/10.1177/ 1090198107313481

Lacunza, A. B., Caballero, S. V., Salazar, R., Sal, J., \& Filgueira, J. (2013). Déficits sociales en adolescentes con sobrepeso y obesidad. Ciencias Psicológicas, 7(1), 25-32.

Larouche, R., Boyer, C., Tremblay, M. S., \& Longmuir, P. (2014). Physical fitness, motor skill, and physical activity relationships in grade 4 to 6 children. Applied Physiology, Nutrition, and Metabolism = Physiologie Appliquee, Nutrition Et Metabolisme, 39(5), 553-559. https://doi.org/10.1139/apnm-2013-0371

Lobstein, T., Jackson-Leach, R., Moodie, M. L., Hall, K. D., Gortmaker, S. L., Swinburn, B. A., ... McPherson, K. (2015). Child and adolescent obesity: part of a bigger picture. The Lancet, 385(9986), 2510-2520. https://doi.org/10.1016/S01406736(14)61746-3

Manchola-González, J., Bagur-Calafat, C., \& Girabent- Farrés, M.
(2017). Fiabilidad de la versión española del cuestionario de actividad física PAQ-C. Revista Internacional de Medicina y Ciencias de la Actividad Física y del Deporte / International Journal of Medicine and Science of Physical Activity and Sport, 17(65). Retrieved from http://www.redalyc.org/ resumen.oa?id=54250121009

Ochoa, G. (2001). Autoestima y percepción del clima escolar en niños con problemas de integración social en el aula. Revista de Psicología General y Aplicada, 54(2), 297-311.

Ortíz, D. C., Cid, M. T., Allepuz, J. P., \& Sánchez, M. L. Z. (2018). Estado actual de la Educación Física desde el punto de vista del profesorado. Propuestas de mejora. (Current state of Physical Education from the point of view of teachers. Improvement proposals). Retos, 0(35), 47-53.

Peèjak, S., Puklek Levpušèek, M., Valenèiè Zuljan, M., Kalin, J., \& Peklaj, C. (2009). Students' Social Behaviour in Relation to their Academic Achievement in Primary and Secondary School: Teacher's Perspective. Psihologijske Teme, 18(1), 55-74.

Piotrowska, P. J., Stride, C. B., Croft, S. E., \& Rowe, R. (2015). Socioeconomic status and antisocial behaviour among children and adolescents: A systematic review and meta-analysis. Clinical Psychology Review, 35, 47-55. https://doi.org/10.1016/ j.cpr.2014.11.003

Ramírez, F. C. (2014). El Test Bull-S Para La Evaluación Sociométrica Del Bullying. Actualización. International Journal of Developmental and Educational Psychology, 7(1), 35-43.

Robins, R. W., Hendin, H. M., \& Trzesniewski, K. H. (2001). Measuring Global Self-Esteem: Construct Validation of a Single-Item Measure and the Rosenberg Self-Esteem Scale , Measuring Global Self-Esteem: Construct Validation of a Single-Item Measure and the Rosenberg Self-Esteem Scale. Personality and Social Psychology Bulletin, 27(2), 151-161. https://doi.org/10.1177/0146167201272002

Rosenberg, M. (2015). Society and the Adolescent Self-Image. Princeton University Press.

Sánchez-Cruz, J.-J., Jiménez-Moleón, J. J., Fernández-Quesada, F., \& Sánchez, M. J. (2013). Prevalencia de obesidad infantil y juvenil en España en 2012. Revista Española de Cardiología, 66(5), 371-376. https://doi.org/10.1016/j.recesp.2012.10.016

Strauss, R. S., Rodzilsky, D., Burack, G., \& Colin, M. (2001). Psychosocial correlates of physical activity in healthy children. Archives of Pediatrics \& Adolescent Medicine, 155(8), 897902.

Strauss, Richard S., \& Pollack, H.A. (2003). Social marginalization of overweight children. Archives of Pediatrics \& Adolescent Medicine, 157(8), 746-752. https://doi.org/10.1001/ archpedi.157.8.746

Tremblay, M. S., Inman, J. W., \& Willms, J. D. (2000). The Relationship between Physical Activity, Self-Esteem, and Academic Achievement in 12-Year-Old Children. Pediatric Exercise Science, 12(3), 312-323. https://doi.org/10.1123/ pes.12.3.312

Vesanto, J., Himberg, J., Alhoniemi, E., \& Parhankangas, J. (1999). Self-organizing map in Matlab: the SOM Toolbox. Proceedings of the Matlab DSP Conference, 99, 16-17.

Wærdahl, R., Nilsen, A. C. E., Svarstad, C., \& Jentoft, N. (2017). Who's at risk? Expanding the categorical understanding of children at risk of social exclusion through measures of selfesteem. Nordic Social Work Research, 7(3), 201-211. https:/ /doi.org/10.1080/2156857X.2016.1277254 